\title{
MDM2 binding protein as a predictor of metastasis and a novel prognostic biomarker in patients with gastric cancer
}

\author{
WEI WANG ${ }^{1-3^{*}}$, ZHONG CHEN $^{3 *}$, JIEJIE JIN ${ }^{1,2^{*}}$, ZIWEN LONG $^{1,2}$, XIAOWEN LIU ${ }^{1,2}$, HONG CAI $^{1,2}$, \\ $\mathrm{YE} \mathrm{ZHOU}^{1,2}$, HUA HUANG ${ }^{1,2}$ and YANONG WANG ${ }^{1,2}$ \\ ${ }^{1}$ Department of Gastric Cancer and Soft Tissue Sarcoma, Fudan University Shanghai Cancer Center, Shanghai; ${ }^{2}$ Department of \\ Oncology, Shanghai Medical College, Fudan University, Shanghai 200032; ${ }^{3}$ Department of Hepatobiliary Surgery, \\ Affiliated Hospital of Nantong University, Nantong, Jiangsu 226000, P.R. China
}

Received October 23, 2015; Accepted April 6, 2017

DOI: $10.3892 / \mathrm{ol} .2017 .7031$

\begin{abstract}
MDM2 binding protein (MTBP) has been revealed to be involved in cancer progression and metastasis. However, the role and clinical implication of MTBP expression in gastric cancer (GC) remains poorly understood. The present study aimed to investigate the clinicopathological significance of MTBP and the prognostic determinant in GC. The expression level of MTBP was examined in cancerous and matched adjacent noncancerous gastric mucosa tissues by reverse transcription-quantitative polymerase chain reaction and western blotting. MTBP expression levels were evaluated by immunohistochemical analysis of tissue microarrays for 352 patients, and association between the expression levels and prognosis in patients with GC were investigated. Kaplan-Meier analysis and Cox's regression models were used to investigate the associations between MTBP expression and prognosis of GC patients. The results of the present study revealed decreased MTBP mRNA $(\mathrm{P}=0.005)$ and protein $(\mathrm{P}=0.001)$ expression levels in tumor tissue compared with in matched adjacent normal tissue mucosa. MTBP expression level in GC was associated with gender $(\mathrm{P}=0.026)$, lymph node metastasis $(\mathrm{P}<0.001)$, distant metastasis $(\mathrm{P}=0.026)$ and pathological tumor-node-metastasis stage $(\mathrm{P}<0.001)$. Kaplan-Meier survival analysis demonstrated that patients with high MTBP expression levels exhibited longer survival times compared with patients with low MTBP expression levels. The multivariate logistic regression analysis
\end{abstract}

Correspondence to: Professor Hua Huang or Professor Yanong Wang, Department of Gastric Cancer and Soft Tissue Sarcoma, Fudan University Shanghai Cancer Center, 270 Dongan Road, Shanghai 200032, P.R. China

E-mail: huahuang@fudan.edu.cn

E-mail: wangyanongsh@gmail.com

*Contributed equally

Key words: MDM2 binding protein, gastric cancer, immunohistochemistry, prognosis revealed that MTBP was independently associated with the presence of lymph node [OR, 0.282; 95\% confidence interval (CI), 0.161-0.494; $\mathrm{P}<0.001]$ and distant metastasis (OR, 0.365; 95\% CI, 0.138-0.965; $\mathrm{P}=0.042)$. Furthermore, the multivariate Cox analysis revealed that low MTBP expression level was significantly associated with longer overall survival time and was recognized as an independent prognostic factor of patient's survival. MTBP expression level was significantly associated with progression and metastasis in GC, suggesting that MTBP may be used as a predictive marker for patient prognosis of GC.

\section{Introduction}

Gastric cancer (GC) is the fourth most common human malignant disease and the second leading cause of cancer-associated mortality worldwide (1-4). Although considerable advances have been made in early diagnosis, surgical techniques and medical treatment, the 5-year survival rate remains poor, particularly in China $(5,6)$. Recurrence and metastasis are the biggest obstacles to the treatment of GC. Therefore, novel prognostic and predictive markers are necessary to improve the assessment of patient outcome and to guide more targeted and individualized therapeutic decisions.

MDM2 binding protein (MTBP), a $104 \mathrm{kDa}$ protein with no known functional motifs, was originally identified as a protein that interacts with the oncoprotein, murine double minute (MDM2), using a yeast two-hybrid screen to bind to the E3 ubiquitin ligase MDM2 (7). MTBP expression can be detected in a wide variety of tissues, with the highest levels of expression in the thymus, testis and ovary. Furthermore, the same tissues exhibited the highest levels of expression of MDM2 (7). A previous study demonstrated that decreased MTBP expression level was associated with tumor metastasis (8). Clinically, loss of MTBP expression is associated with reduced survival of patients with head and neck carcinoma (9). Overexpression of MTBP protects MDM2 from self-ubiquitination, which induces MDM2 stabilization and p53 degradation (10). MDM2 is a major negative regulator of the tumor suppressor p53 (11,12). p53, a potent growth suppressive and proapoptotic molecule, may serve a key role in cell-cycle regulation and be central to protecting cells from uncontrolled growth $(13,14)$. 
p53 is also the most frequently altered protein in human cancer (15). In total, $\sim 50 \%$ of all human malignancies harbor mutations or deletions in the p53 gene that disable the tumor suppressor function of the encoded protein $(16,17)$. A subsequent study revealed that MTBP regulated $\mathrm{p} 53$ via modulation of MDM2 ubiquitin ligase activity (10). Boyd et al (7) reported that overexpression of MTBP induced $\mathrm{G}_{1}$ arrest of the cell cycle independent of $\mathrm{p} 53$. MTBP contributed to $\mathrm{p} 53 / \mathrm{MDM} 2$ homeostasis and generated MTBP-reactive antisera, and MTBP phenotypes have been identified to be compatible with potentially oncogenic and tumor suppressive activities for this gene $(7,10)$. In particular, overexpression of MTBP in tumor cell lines increased MDM2 ubiquitin ligase activity, which induced p53 degradation, whereas suppression of MTBP expression had the opposite effect $(7,10)$. MTBP has been involved in tumor progression of patients with head and neck cancer; it may predict disease progression for patients with squamous cell carcinoma of the head and neck (9).

The present study evaluated MTBP expression level in gastric tissue microarrays (TMA) by immunohistochemistry (IHC) and its clinical significances. The results demonstrated the crucial role of MTBP as a prognostic and metastatic marker as well as a novel potential therapeutic target in GC.

\section{Materials and methods}

Patient selection and tissue specimens. Tissue samples were obtained from 352 patients with GC who underwent surgical resection at the Department of Gastric Cancer and Soft Tissue Sarcoma, Fudan University Shanghai Cancer Center from April 2010 to April 2011. No preoperative chemotherapy or radiotherapy had been performed in any of these cases. In the present study, there were 275 men and 77 women with a mean age of 58 years (range, 32-88 years). The clinical data of patients were obtained from the medical record of Fudan University Shanghai Cancer Center (Shanghai, China). Ethical approval for human subjects was obtained from the Research Ethics Committee of Fudan University Shanghai Cancer Center, and written informed consent was obtained from all patients prior to enrollment in the present study. One pair of tissue samples was obtained from each of the 352 patients. All patients were regularly followed-up by telephone. The duration of follow-up was defined as the interval from the date of the treatment to the date of mortality or the final follow-up, with the final follow-up being the 15th of December 2014. The clinicopathological characteristics of patients in the TMA are summarized in Table I. Tumor size was defined as the maximum diameter of the tumor. Tumor-node-metastasis (TNM) classification of gastric carcinoma was based on the 7th edition of the American Joint Committee on Cancer staging system (18). Borrman type was classified as previously described (19). Histological grade was classified according to the Japanese Classification of Gastric Carcinoma as well, moderately and poorly differentiated (20). Preoperative carcinoembryonic antigen (CEA) levels were obtained using an ELISA kit according to the manufacturer's protocol (cat no. ab99992; Abcam, Cambridge, UK). Briefly, the assay employed anti-human CEA antibody coated onto a 96-well plate. Standard or serum samples were pipetted into the wells and CEA present in samples was bound to the wells by the immobilized antibody. The wells were washed and biotinylated anti-human CEA antibody was added. Following the washing of unbound biotinylated antibody, horseradish peroxidase-conjugated streptavidin was pipetted into the wells. The wells were washed again, tetramethylbenzidine substrate solution was added and color developed in proportion to the amount of bound CEA. Absorbance value was measured at $450 \mathrm{~nm}$. CEA concentration in serum was achieved according to standard curves.

TMA construction. TMAs were constructed as described previously (21). In brief, identical $1.5 \mathrm{~mm}$ diameter cylinders from the center of the tumor were included from each case. Cylinders from the donor blocks were transferred to the recipient paraffin block at defined array positions. Consecutive $4 \mathrm{~mm}$ sections were placed on 3-aminopropyltriethoxysilane coated slides (in collaboration with Shanghai Biochip Company, Shanghai, China). Hematoxylin and eosin-stained slides from each tissue block were reviewed by a senior consultant pathologist, and performed according to a previously described protocol (22).

Immunohistochemical staining. IHC was performed as previously described (23). Sections $(4 \mu \mathrm{m})$ of primary gastric adenocarcinoma and the adjacent normal gastric tissues were subjected to immunohistochemical staining as follows: Formalin-fixed and paraffin-embedded tissue sections were deparaffinized in xylene, rehydrated in a graded alcohol series and washed with PBS. Subsequently, the tissue sections were immersed in $10 \mathrm{mmol} / \mathrm{l}$ citrate buffer $(\mathrm{pH} \mathrm{6.0)}$ ) and heated in a microwave for $30 \mathrm{~min}$. Following cooling to room temperature, endogenous peroxidase was blocked by incubation with $3 \% \mathrm{H}_{2} \mathrm{O}_{2}$ in methanol $\left(36^{\circ} \mathrm{C}, 60 \mathrm{~min}\right)$. Nonspecific binding was blocked by incubating the tissue sections with $1 \%$ bovine serum albumin (Sigma-Aldrich; Merck KGaA, Darmstadt, Germany) in a humid chamber for $60 \mathrm{~min}$ at $4^{\circ} \mathrm{C}$. Incubation with the primary antibodies was subsequently performed overnight at $4^{\circ} \mathrm{C}$ using mouse anti-human MTBP (dilution, 1:200; cat no. sc-99047; Santa Cruz Biotechnology, Inc., Dallas, TX, USA). The negative controls were treated identically but with the primary antibodies omitted. Subsequently, incubation with suitable secondary antibodies (cat no. pk-6100; Vector Laboratories, Burlingame, CA, USA) at room temperature. Finally, diaminobenzidine (Vector Laboratories) was used for signal development, and the sections were counterstained with $20 \%$ hematoxylin for $8 \mathrm{~min}$ at room temperature. Detection was performed using a Dako EnVision system (Dako; Agilent Technologies, Inc., Santa Clara, CA, USA).

Immunohistochemical evaluation. The expression level of MTBP in tumor and normal adjacent tissues was assessed by two investigators blinded to the clinical data and to the other investigator's score. The scores were determined by the proportion of positive tumor cells and the intensity of the coloring for MTBP, as described previously (9). MTBP cytoplasmic expression level was initially assigned an intensity grade: 0 , no staining; 1, weak staining; 2 , moderate staining; and 3, strong staining. Nuclear MTBP staining was scored positive or negative. The scores were categorized into 2 groups: Low expression ( $0 / 1$ cytoplasmic staining) with no nuclear staining and high expression (2/3 cytoplasmic staining) and/or nuclear positivity. 
Table I. Association between MTBP expression levels and clinicopathological characteristics of gastric cancer.

\begin{tabular}{|c|c|c|c|c|}
\hline \multirow[b]{2}{*}{ Variable } & \multirow[b]{2}{*}{ Patients, n (\%) } & \multicolumn{2}{|c|}{ MTBP expression level, n (\%) } & \multirow[b]{2}{*}{ P-value } \\
\hline & & Low & High & \\
\hline All cases & $352(100)$ & $271(77.0)$ & $81(23.0)$ & \\
\hline Gender & & & & 0.026 \\
\hline Male & $275(78.1)$ & $219(79.6)$ & $56(20.4)$ & \\
\hline Female & $77(21.9)$ & $52(67.5)$ & $25(32.5)$ & \\
\hline Age, years & & & & 0.234 \\
\hline$\leq 60$ & $155(44.0)$ & $124(80.0)$ & $31(20.0)$ & \\
\hline$>60$ & $197(56.0)$ & $147(74.6)$ & $50(25.4)$ & \\
\hline Tumor size, $\mathrm{cm}$ & & & & 0.077 \\
\hline$<5$ & $187(53.1)$ & $137(73.3)$ & $50(26.7)$ & \\
\hline$\geq 5$ & $165(46.9)$ & $134(81.2)$ & $31(18.8)$ & \\
\hline Borrmann type & & & & 0.075 \\
\hline I & $20(5.70)$ & $14(70.0)$ & $6(30.0)$ & \\
\hline II & $17(4.80)$ & $9(52.9)$ & $8(47.1)$ & \\
\hline III & $302(85.8)$ & $237(78.5)$ & $65(21.5)$ & \\
\hline IV & $13(3.70)$ & $11(84.6)$ & $2(15.4)$ & \\
\hline Depth of invasion & & & & 0.171 \\
\hline $\mathrm{T} 1$ & $12(3.40)$ & $8(66.7)$ & $4(33.3)$ & \\
\hline $\mathrm{T} 2$ & $16(4.50)$ & $10(62.5)$ & $6(37.5)$ & \\
\hline $\mathrm{T} 3$ & $27(7.70)$ & $18(66.7)$ & $9(33.3)$ & \\
\hline $\mathrm{T} 4$ & $297(84.4)$ & $235(79.1)$ & $62(20.9)$ & \\
\hline Lymph node metastasis & & & & $<0.001$ \\
\hline No & $79(22.4)$ & $45(57.0)$ & $34(43.0)$ & \\
\hline N1 & $69(19.6)$ & $54(78.3)$ & $15(21.7)$ & \\
\hline $\mathrm{N} 2$ & $65(18.5)$ & $55(84.6)$ & $10(15.4)$ & \\
\hline N3 & $139(39.5)$ & $117(84.2)$ & $22(15.8)$ & \\
\hline Distant metastasis & & & & 0.026 \\
\hline M0 & $304(86.4)$ & $228(75.0)$ & $76(25.0)$ & \\
\hline M1 & $48(13.6)$ & $43(89.6)$ & $5(10.4)$ & \\
\hline pTNM stage & & & & $<0.001$ \\
\hline I & $11(3.10)$ & $7(63.6)$ & $4(36.4)$ & \\
\hline II & $72(20.5)$ & $38(52.8)$ & $34(47.2)$ & \\
\hline III & $221(62.8)$ & $183(82.8)$ & $38(17.2)$ & \\
\hline IV & 48 (13.6) & $43(89.6)$ & $5(10.4)$ & \\
\hline Histological grade & & & & 0.441 \\
\hline Well & $18(5.10)$ & $12(66.7)$ & $6(33.3)$ & \\
\hline Moderately & $159(45.2)$ & $126(79.2)$ & $33(20.8)$ & \\
\hline Poorly & $175(49.7)$ & $133(76.0)$ & $42(24.0)$ & \\
\hline Preoperative CEA & & & & 0.113 \\
\hline Elevated & $84(24.1)$ & $70(83.3)$ & $14(16.7)$ & \\
\hline Normal & $268(75.9)$ & $201(75.0)$ & $67(25.0)$ & \\
\hline
\end{tabular}

pTNM, pathological tumor-node-metastasis; CEA, carcinoembryonic antigen; MTBP, MDM2 binding protein.

Western blotting. The reagents and protocols used were described in detail previously (24). Paired tumor tissues and surrounding non-tumor tissues were ground to powder in liquid nitrogen and lysed with SDS-PAGE sample buffer. Primary antibodies against MTBP were purchased from
Abcam (1:500; cat no. ab115529) and used according to the manufacturer's instructions. A monoclonal antibody against GAPDH (1:1,000; cat no. sc-69778; Santa Cruz Biotechnology, Inc.) was used as an internal control. GAPDH was used as a control. The primary antibodies were detected with 
A

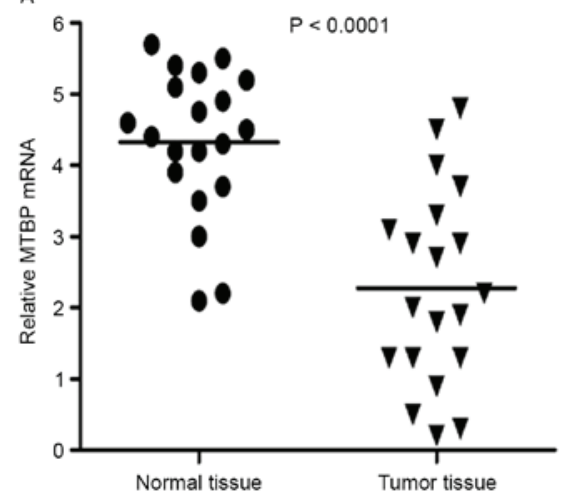

B
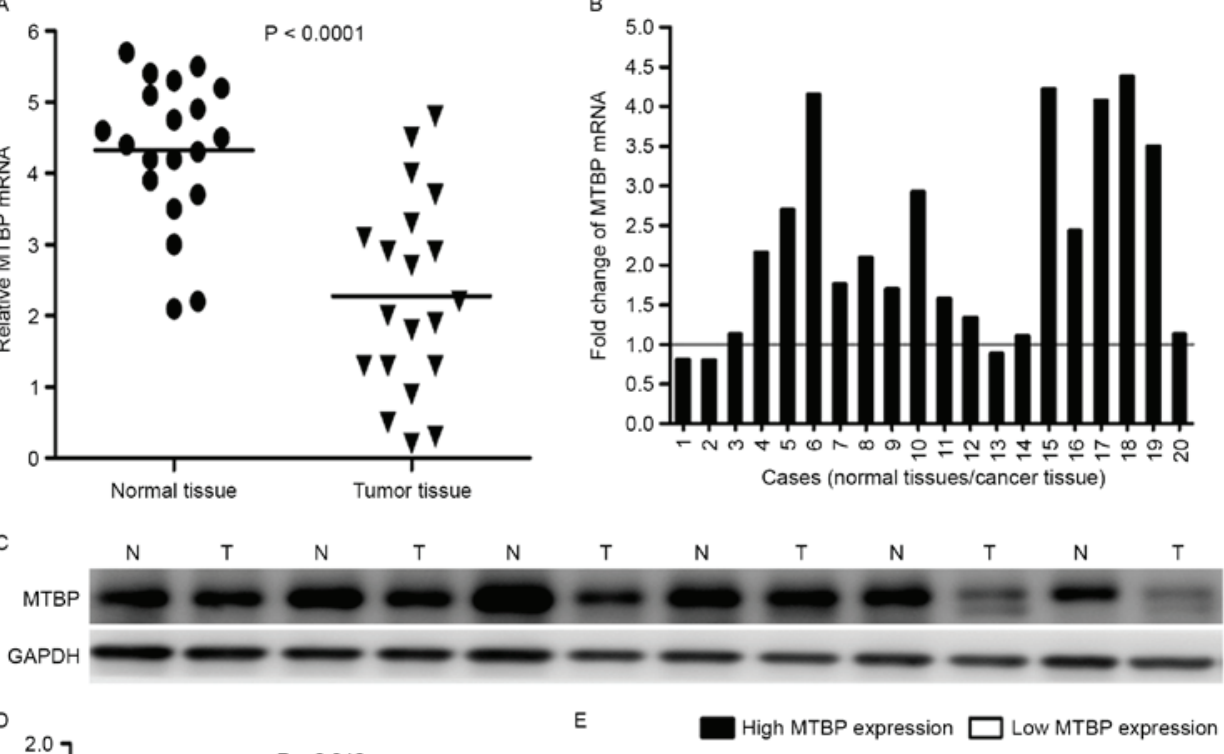

D
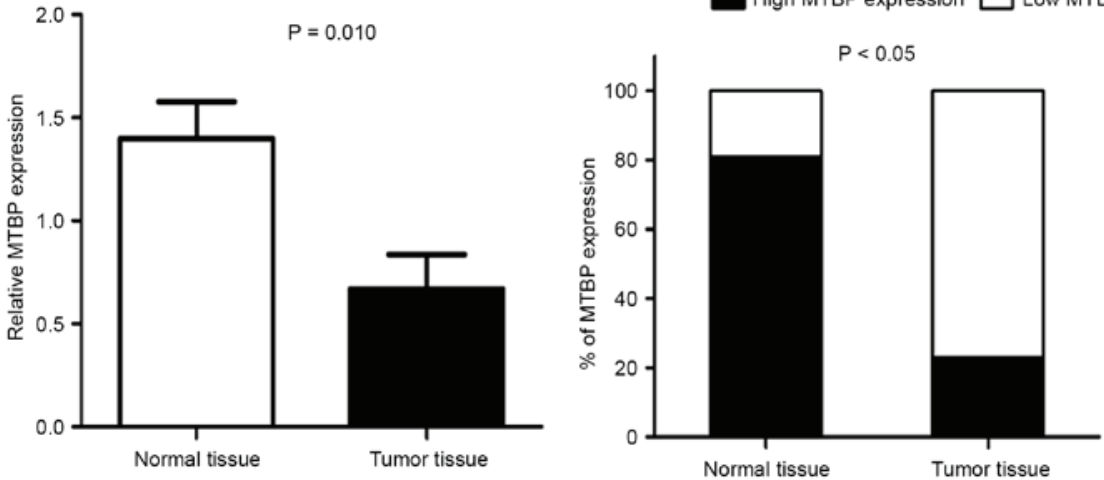

Figure 1. Expression levels of MTBP in gastric tumor and adjacent normal tissues. (A) The average relative mRNA expression levels of MTBP in GC tumor tissues compared with in the paired adjacent normal tissues $(\mathrm{P}<0.0001)$. (B) The fold change of MTBP expression levels in gastric tumor tissues compared with in paired adjacent normal tissues evaluated by reverse transcription-quantitative polymerase chain reaction and normalized to GAPDH. (C) Western blotting analysis of MTBP expressed levels in 6 paired representative gastric tumor tissues and adjacent normal tissues. (D) The relative expression levels of MTBP in gastric tumor and adjacent normal tissues. (E) Percentages of samples displaying low or high expression levels of MTBP relative to tumor tissues and adjacent normal tissues. T, tumor tissues; N, normal tissues; GC, gastric cancer; MTBP, MDM2 binding protein.

horseradish peroxidase-conjugated secondary antibodies (1:1,000; cat no. sc-2351; Santa Cruz Biotechnology, Inc.); Immunoreactive bands were visualized using SuperSignal West Pico Chemiluminescent Substrate (cat no. NCI5080; Pierce; Thermo Fisher Scientific, Inc., Waltham, MA, USA). The gray value of each band was measured and data are presented as a ratio to GAPDH (ImageJ 1.46; National Institutes of Health, Bethesda, MD, USA). Each experiment was repeated $\geq 3$ times.

Reverse transcription-quantitative polymerase chain reaction $(R T-q P C R)$. As described previously (25), total RNA was extracted from tumor and normal adjacent tissues using TRIzol ${ }^{\circledR}$ reagent (Invitrogen; Thermo Fisher Scientific, Inc.). RT-qPCR analysis was performed using an ABI PRISM 7500 Real-Time PCR system (Applied Biosystems; Thermo Fisher Scientific, Inc.). Each well (20 $\mu 1$ reaction volume) contained $10 \mu 1$ Power SYBR-Green PCR Master Mix (Applied Biosystems; Thermo Fisher Scientific, Inc.), $1 \mu$ l of each primer ( $5 \mu \mathrm{mol} / \mathrm{l})$ and $1 \mu \mathrm{l}$ template. The following primers were used: MTBP forward, 5'-TCCTGTAGTTTCGTCAGATCCT-3' and reverse, 5'-CCGTTTCAATCGGGATACTTCA-3'. GAPDH forward, 5'-ACAGCCTCAAGATCATCAGCA-3' and reverse, 5'-ATGAGTCCTTCCACGATACCA-3'. GAPDH was used as internal control. Quantification was performed as described previously (25).

Statistical analyses. All statistical analyses were performed using SPSS version 19.0 for Windows (IBM Corp., Armonk, NY, USA). The associations between MTBP expression level and clinicopathological characteristics were evaluated using the $\chi^{2}$ test. Cumulative survival time was determined by the Kaplan-Meier method using GraphPad Prism software (version 5.0; GraphPad Software, Inc., La Jolla, CA, USA), and differences in survival curves were analyzed using the log-rank test. Multivariate logistic regressions were used to assess the association between MTBP expression level and metastasis. Multivariate analysis was performed using the Cox's proportional hazards regression model on all significant characteristics evaluated for univariate analysis. $\mathrm{P}<0.05$ was considered to indicate a statistically significant difference.

\section{Results}

MTBP $m R N A$ and protein expression levels in GC tissues and normal adjacent tissues. Representative data are presented in Fig. 1. RT-qPCR and western blot analysis were used to confirm 


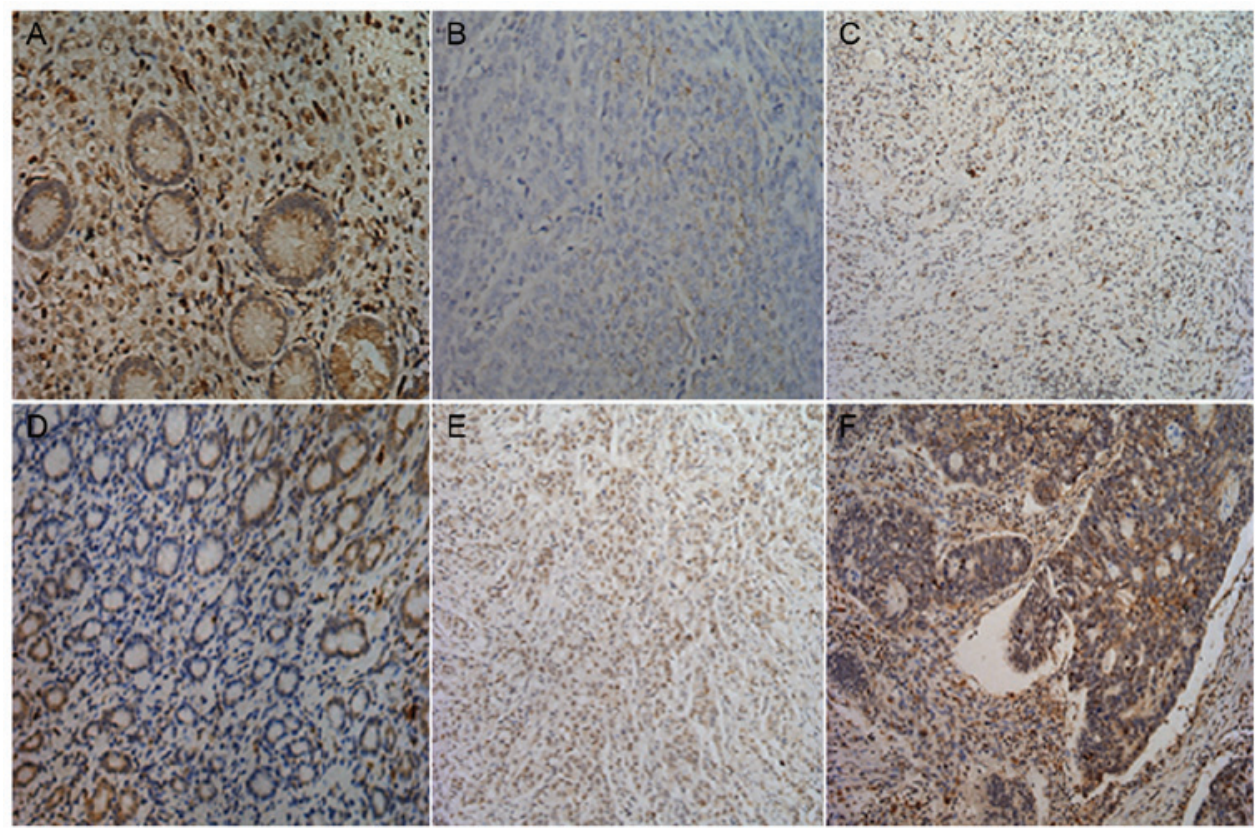

Figure 2. Representative images of MTBP immunohistochemical staining in gastric tumor tissues and adjacent normal tissues. (A) High expression levels of MTBP in normal mucosa. (B) No cytoplasmic staining in tumor tissue. (C) Weak cytoplasmic staining with positive nuclear MTBP staining in tumor tissue. (D) Low expression level of MTBP in normal mucosa. (E) Moderate cytoplasmic staining with nuclear MTBP staining in tumor tissue. (F) Strong cytoplasmic staining with absence of nuclear staining (original magnification, x200). MTBP, MDM2 binding protein.

Table II. Immunohistochemical evaluation of nuclear and cytoplasmic staining.

\begin{tabular}{lrl}
\hline Staining & Number & \multicolumn{1}{c}{ Intensity } \\
\hline Cytoplasmic & 122 & No staining \\
0 & 149 & Weak staining \\
1 & 47 & Moderate staining \\
2 & 34 & Strong staining \\
3 & & \\
Nuclear & 81 & Nuclear staining \\
Positive & 271 & No nuclear staining \\
Negative & & \\
Combined scoring & 271 & 0/1 cytoplasmic staining + \\
Low expression & & no nuclear staining \\
& 81 & 2/3 cytoplasmic staining + \\
High expression & & nuclear positivity \\
&
\end{tabular}

mRNA and protein expression levels. The mean expression level of MTBP mRNA in normal tissues was significantly higher compared with in tumor tissues $(\mathrm{P}<0.0001$; Fig. 1A). Furthermore, the MTBP mRNA expression level was significantly lower in 17/20 (85\%) GC tissues compared with in the matched adjacent normal gastric mucosa tissues (Fig. 1B).

The difference in MTBP expression levels between tumors and normal tissues revealed at the protein level was investigated by western blotting. The representative western blotting results in six cases are presented in (Fig. 1C). The relative level of MTBP expression was normalized to the GAPDH of the same samples. Western blot analysis demonstrated that the expression level of MTBP protein was markedly decreased in $10 / 14(71.4 \%)$ gastric tumor tissues compared with in the corresponding adjacent normal tissues, which was consistent with that of the RT-qPCR results. The average MTBP protein expression level in 14 GC tissues was significantly lower compared with in the matched adjacent normal gastric mucosa tissues $(\mathrm{P}<0.05)$ (Fig. 1D). Fig. 1E revealed that normal tissues demonstrated higher expression levels of MTBP (81\%), statistical analysis revealed a lower expression level of MTBP in gastric tumors (19\%) compared with in adjacent normal tissues $(\mathrm{P}<0.05)$.

Association between MTBP expression level and clinicopathological characteristics in GC. To elucidate the biological significance of MTBP in GC, the present study examined the immunohistochemical expression levels of MTBP in GC tissues (Fig. 2). MTBP staining was mainly located in the cytoplasm and/or the nucleus of tumor cells, as presented in Table II, consistent with that of a previous study (9). As presented in Table I, there were no significant associations between the expression level of MTBP protein and age, tumor size, Borrmann type, depth of invasion, histological grade or preoperative carcinoembryonic antigen expression levels in patients with GC. However, the expression level of MTBP was significantly associated with gender, lymph node metastasis, distant metastasis and pathological tumor-node-metastasis (pTNM) stage in GC tissues.

Predictive significance of MTBP expression level in $G C$ with distant and lymph node metastases. It was revealed that low MTBP expression level was associated with higher distant metastasis and lymph node metastases rate (Fig. 3). A multivariate logistic regression analysis was performed to evaluate the independently predictive significance of 
Table III. Associations of MTBP expression level with distant and lymph node metastasis.

\begin{tabular}{|c|c|c|c|c|c|}
\hline Variable & $\mathrm{B}$ & SE & OR & $95 \% \mathrm{CI}$ & P-value \\
\hline \multicolumn{6}{|l|}{ Distant metastasis, $\mathrm{n}=48$} \\
\hline Age, years $(\leq 60$ vs. $>60)$ & 0.025 & 0.320 & 1.025 & $0.547-1.921$ & 0.938 \\
\hline Tumor size (<5 vs. $\geq 5 \mathrm{~cm})$ & 0.664 & 0.323 & 1.942 & $1.031-3.659$ & 0.040 \\
\hline Differentiation (well/moderately vs. poorly) & -0.402 & 0.323 & 0.669 & $0.355-1.259$ & 0.213 \\
\hline Preoperative CEA (elevated vs. normal) & 0.051 & 0.362 & 1.052 & $0.512-2.163$ & 0.890 \\
\hline MTBP expression (high vs. low) & -1.007 & 0.496 & 0.365 & $0.138-0.965$ & 0.042 \\
\hline \multicolumn{6}{|l|}{ Lymph node metastasis, $n=273$} \\
\hline Age, years $(\leq 60$ vs. $>60)$ & -0.369 & 0.276 & 0.691 & $0.402-1.188$ & 0.182 \\
\hline Tumor size (<5 vs. $\geq 5 \mathrm{~cm})$ & 0.500 & 0.275 & 1.649 & $0.962-2.826$ & 0.690 \\
\hline Differentiation (well/moderately vs. poorly) ${ }^{\mathrm{a}}$ & -0.344 & 0.271 & 0.709 & $0.417-1.206$ & 0.204 \\
\hline Preoperative CEA (elevated vs. normal) & -0.112 & 0.314 & 0.894 & $0.483-1.653$ & 0.720 \\
\hline MTBP expression (high vs. low) & -1.265 & 0.285 & 0.282 & $0.161-0.494$ & $<0.001$ \\
\hline
\end{tabular}

${ }^{a}$ According to the Japanese Classification of Gastric Carcinoma. CEA, carcinoembryonic antigen; MTBP, MDM2 binding protein; B, the parameter estimator of association coefficient; SE, standard error of B; OR, odds ratio; 95\% CI, 95\% confidence interval.
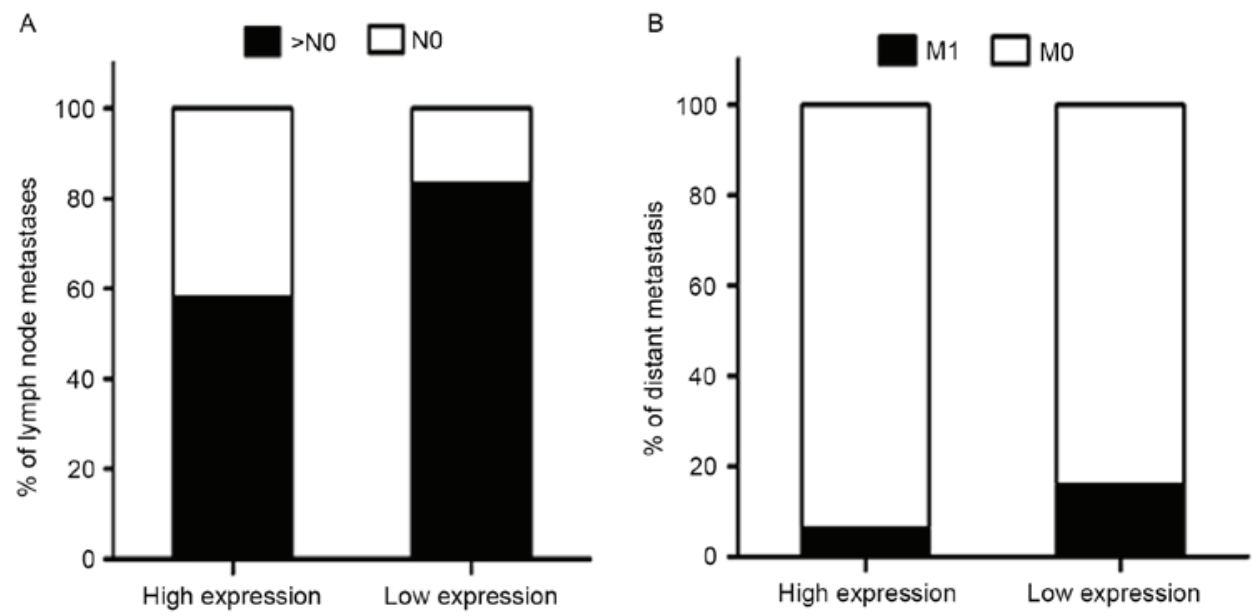

Figure 3. Low expression levels of MTBP demonstrated higher (A) lymph node metastases and (B) distant metastasis rates. MTBP, MDM2 binding protein. N0, without lymph node metastasis; >NO, with lymph node metastases; M0, without distant metastasis; M1, with distant metastasis.

MTBP expression level for distant and lymph node metastases (Table III). The results demonstrated that the tumor size [OR, 1.942; 95\% confidence interval (CI), 1.031-3.659; $\mathrm{P}=0.040]$ and MTBP expression level (OR, 0.365; 95\% CI, $0.138-0.965 ; \mathrm{P}=0.042$ ) were independently associated with distant metastasis. As described above, the multivariate logistic regression analysis revealed that MTBP expression level was significantly associated with the presence of lymph node metastasis (OR, 0.282; 95\% CI, 0.161-0.494; $\mathrm{P}<0.001)$ (all Table III).

Prognostic significance of MTBP expression level in $G C$ tissues. To investigate the prognostic value of MTBP expression level in patients with GC, overall survival (OS) analysis was performed in 352 patients with GC. The 1- and 3-year OS rates were 88.1 and $72.4 \%$, respectively. The OS rate of the high-level expression group was significantly longer compared with that of the low-level expression group (Fig. 4).
Univariate and multivariate analyses were performed to determine the predictors for OS (Table IV). To further determine the effect of MTBP expression on OS, the present study first performed univariate analysis of traditional clinicopathological variables for prognosis. In univariate analysis, low expression level of MTBP $(\mathrm{P}=0.017)$, larger tumor size $(\mathrm{P}=0.001)$, lymph node metastasis $(\mathrm{P}=0.001)$, elevated preoperative CEA $(\mathrm{P}=0.047)$ and TNM stage $(\mathrm{P}<0.001)$ were revealed to be associated with a poor OS rate of patients with GC. Furthermore, to evaluate the independent impact of MTBP expression level on OS, a multivariate Cox's regression model was performed. The results demonstrated that low MTBP expression level (HR, 0.633; 95\% CI, 0.417-0.961) was a poor independent prognostic factor for OS in patients with GC. In addition, tumor size (HR, 0.582; 95\% CI, 0.389-0.871) and TNM stage (HR, 2.720; 95\% CI, 1.395-5.305) revealed independent prognostic value in the multivariate analysis (Table IV). 
Table IV. Univariate and multivariate survival analyses in patients with gastric cancer.

\begin{tabular}{|c|c|c|c|c|c|c|}
\hline \multirow[b]{2}{*}{ Independent factors } & \multicolumn{3}{|c|}{ Univariate analysis } & \multicolumn{3}{|c|}{ Multivariate analysis } \\
\hline & HR & $95 \% \mathrm{CI}$ & P-value & HR & $95 \% \mathrm{CI}$ & P-value \\
\hline Gender (male vs. female) & 1.099 & $0.629-1.619$ & 0.970 & & & \\
\hline Age, years ( $\leq 60$ vs. $>60)$ & 0.992 & $0.669-1.473$ & 0.969 & & & \\
\hline Tumor size, $\mathrm{cm}(<5$ vs. $\geq 5)$ & 0.520 & $0.349-0.777$ & 0.001 & 0.582 & $0.389-0.871$ & 0.008 \\
\hline Borrmann type (I/II vs. III/IV) & 1.293 & $0.652-2.565$ & 0.462 & & & \\
\hline pTNM stage (I/II vs. III/IV) & 3.233 & $1.682-6.214$ & $<0.001$ & 2.720 & $1.395-5.305$ & 0.003 \\
\hline Differentiation (well/moderate vs. poor) ${ }^{\mathrm{a}}$ & 1.444 & $0.972-2.145$ & 0.069 & & & \\
\hline Preoperative CEA (elevated vs. normal) & 1.719 & $1.007-2.934$ & 0.047 & 1.290 & $0.749-2.222$ & 0.359 \\
\hline MTBP expression level High vs. low & 0.602 & $0.397-0.913$ & 0.017 & 0.633 & $0.417-0.961$ & 0.032 \\
\hline
\end{tabular}

${ }^{a}$ According to the Japanese Classification of Gastric Carcinoma. pTNM, pathological tumor-node-metastasis; CEA, carcinoembryonic antigen; MTBP, MDM2 binding protein; HR, hazard ratio; 95\% CI, 95\% confidence interval.

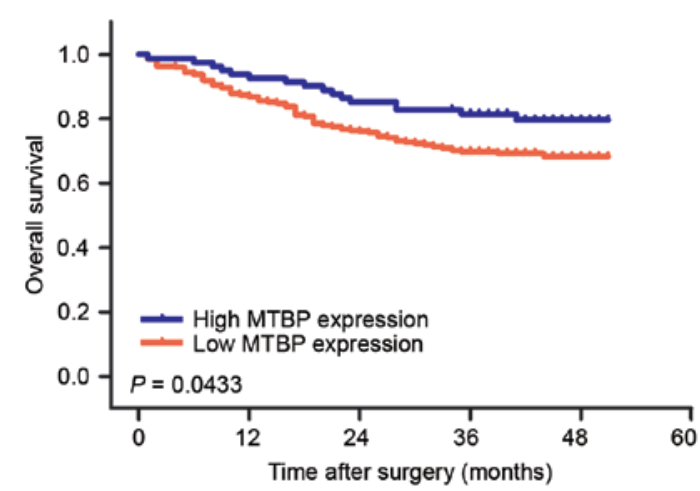

Figure 4. Kaplan-Meier curves for overall survival in patients with gastric cancer with low and high expression levels of MTBP. MTBP, MDM2 binding protein.

\section{Discussion}

MTBP is a protein that interacts with MDM2, a major inhibitor of the tumor suppressor p53. p53 was identified as a tumor suppressor protein and is the most commonly mutated gene in human cancer $(14,16)$. Alam et al $(26)$ revealed that the impact of this particular protein MTBP is to stabilize the steady state expression level of MDM2.

MTBP is a cellular protein and the expression levels have been found to be higher in cancer cells $(8,9)$. It has been established that MTBP serves a role in the suppression of tumor progression, and MTBP has been considered to be a biomarker for various types of cancer. However, the role of MTBP in GC remains unknown. To the best of our knowledge, the present study is the first attempt to elucidate the role of MTBP in GC. The present study revealed that MTBP expression level was reduced in GC tissues compared with in matched non-tumor tissues, which implied that MTBP may be a candidate tumor suppressor molecule in GC. Furthermore, the present study investigated the expression level of MTBP in 352 GC tissues by IHC. It was demonstrated that MTBP was significantly associated with gender, lymph node metastasis, distant metastasis and pTNM stage. Agarwal et al (27) revealed that overexpression of MTBP in osteosarcoma cells suppressed metastasis with little effect on primary tumor growth in an orthotopic mouse model, and that MTBP inhibited cell migration and filopodia formation. The present study demonstrated that MTBP expression level was significantly associated with the OS rate of patients with GC. Kaplan-Meier analysis of OS revealed that a unique cohort of patients with GC with low MTBP expression level exhibited significantly poorer OS rate, indicating that low MTBP expression level was a marker of poor prognosis for patients with GC. Furthermore, Cox proportional hazards model revealed that MTBP status was an independent prognostic predictor for patients with GC. Vlatkovic et al (9) demonstrated that loss of MTBP expression was associated with reduced survival of patients with head and neck squamous cell carcinoma and served as an independent prognostic factor. These observations indicated that MTBP expression levels may serve as a powerful novel prognosticator of GC. The TMA immunohistochemical assay of the present study supported this hypothesis, as the TMA results and clinical data revealed that MTBP exhibited a positive impact on patient outcomes. Thus, MTBP may constitute a molecular prognostic marker for patients with GC, identifying the individuals who are more likely to have a higher risk of mortality. To the best of our knowledge, this is the first study to demonstrate the positive role of MTBP in patients with GC. However, this present retrospective cohort study was limited to relatively small case series. Therefore, further validation is required.

In conclusion, the present study determined that MTBP serves a critical role in the suppression of tumor progression and metastasis formation of GC. MTBP expression levels demonstrated significant associations with tumor metastasis. These findings suggested that MTBP may be a potential prognostic marker for GC, and possibly an individual therapeutic target.

\section{Acknowledgements}

The present study was supported by the Natural Science Foundation of China (grant no. 81272726), the Specialized 
Research Fund for the Doctoral Program of Higher Education (grant no. 20110071120097) and Shanghai Municipal Health Bureau Research Project (grant no. 20114174).

\section{References}

1. González CA, Sala N and Rokkas T: Gastric cancer: Epidemiologic aspects. Helicobacter 18 (Suppl 1): S34-S38, 2013.

2. Wadhwa R, Song S, Lee JS, Yao Y, Wei Q and Ajani JA: Gastric cancer-molecular and clinical dimensions. Nat Rev Clin Oncol 10: 643-655, 2013

3. Siegel R, Naishadham D and Jemal A: Cancer statistics, 2012. CA Cancer J Clin 62: 10-29, 2012.

4. Peleteiro B, Bastos A, Ferro A and Lunet N: Prevalence of Helicobacter pylori infection worldwide: A systematic review of studies with national coverage. Dig Dis Sci 59: 1698-1709, 2014.

5. Kamangar F, Dores GM and Anderson WF: Patterns of cancer incidence, mortality, and prevalence across five continents: Defining priorities to reduce cancer disparities in different geographic regions of the world. J Clin Oncol 24: 2137-2150, 2006.

6. Hartgrink HH, Jansen EP, van Grieken NC and van de Velde CJ: Gastric cancer. Lancet 374: 477-490, 2009.

7. Boyd MT, Vlatkovic N and Haines DS: A novel cellular protein (MTBP) binds to MDM2 and induces a G1 arrest that is suppressed by MDM2. J Biol Chem 275: 31883-31890, 2000.

8. Iwakuma T, Tochigi Y, Van Pelt CS, Caldwell LC, Terzian T, Parant JM, Chau GP, Koch JG, Eischen CM and Lozano G: Mtbp haploinsufficiency in mice increases tumor metastasis. Oncogene 27: 1813-1820, 2008.

9. Vlatković N, El-Fert A, Devling T, Ray-Sinha A, Gore DM, Rubbi CP, Dodson A, Jones AS, Helliwell TR, Jones TM and Boyd MT: Loss of MTBP expression is associated with reduced survival in a biomarker-defined subset of patients with squamous cell carcinoma of the head and neck. Cancer 117: 2939-2950, 2011.

10. Brady M, Vlatkovic N and Boyd MT: Regulation of p53 and MDM2 activity by MTBP. Mol Cell Biol 25: 545-553, 2005.

11. Iwakuma T and Lozano G: MDM2, an introduction. Mol Cancer Res 1: 993-1000, 2003.

12. Marine JC and Lozano G: Mdm2-mediated ubiquitylation: p53 and beyond. Cell Death Differ 17: 93-102, 2010.

13. Carson DA and Lois A: Cancer progression and p53. Lancet 346: 1009-1011, 1995.
14. Lane DP: Cancer. p53, guardian of the genome. Nature 358 15-16, 1992.

15. Vassilev LT: p53 Activation by small molecules: Application in oncology. J Med Chem 48: 4491-4499, 2005.

16. Hollstein M, Sidransky D, Vogelstein B and Harris CC: p53 mutations in human cancers. Science 253: 49-53, 1991

17. Hainaut $P$ and Hollstein M: $p 53$ and human cancer: The first ten thousand mutations. Adv Cancer Res 77: 81-137, 2000.

18. Sobin LH, Gospodarowicz MK and Wittekind C: TNM Classification of Malignant Tumors. 7th edition. Wiley-Liss, New York, NY, 2009.

19. Borrmann R: Geschwülste des Magens und Duodenums. In: Verdauungsschlauch. Erster Teil Rachen und Tonsillen, Speiseröhre Magen und Darm, Bauchfell. Borchardt $\mathrm{H}$, Borrmann R, Christeller E, et al (eds). Vienna, Springer, pp812-1054, 1926.

20. Sano T and Aiko T: New Japanese classifications and treatment guidelines for gastric cancer: Revision concepts and major revised points. Gastric Cancer 14: 97-100, 2011.

21. Simon R, Mirlacher M and Sauter G: Tissue microarrays. Biotechniques 36: 98-105, 2004.

22. Zhou Z, Chen ZW, Yang XH, Shen L, Ai XH, Lu S and Luo QQ: Establishment of a biomarker model for predicting bone metastasis in resected stage III non-small cell lung cancer. J Exp Clin Cancer Res 31: 34, 2012.

23. Ma Q, Li P, Xu M, Yin J, Su Z, Li W and Zhang J: Ku80 is highly expressed in lung adenocarcinoma and promotes cisplatin resistance. J Exp Clin Cancer Res 31: 99, 2012.

24. Fan C, Miao Y, Zhang X, Liu D, Jiang G, Lin X, Han Q, Luan L, $\mathrm{Xu} \mathrm{Z}$ and Wang E: Btbd7 contributes to reduced E-cadherin expression and predicts poor prognosis in non-small cell lung cancer. BMC Cancer 14: 704, 2014.

25. Ji K, Ye L, Ruge F, Hargest R, Mason MD and Jiang WG: Implication of metastasis suppressor gene, Kiss-1 and its receptor Kiss-1R in colorectal cancer. BMC Cancer 14: 723, 2014.

26. Alam MJ, Fatima N, Devi GR, Ravins and Singh RK: The enhancement of stability of p53 in MTBP induced p53-MDM2 regulatory network. Biosystems 110: 74-83, 2012.

27. Agarwal N, Adhikari AS, Iyer SV, Hekmatdoost K, Welch DR and Iwakuma T: MTBP suppresses cell migration and filopodia formation by inhibiting ACTN4. Oncogene 32: 462-470, 2013. 